\title{
Optimal Placement of a UAV to Maximize the Lifetime of Wireless Devices
}

\author{
Hazim Shakhatreh \\ Department of Electrical and \\ Computer Engineering \\ New Jersey Institute of Technology \\ Email: hms35@njit.edu
}

\author{
Abdallah Khreishah \\ Department of Electrical and \\ Computer Engineering \\ New Jersey Institute of Technology \\ Email: abdallah@njit.edu
}

\begin{abstract}
Unmanned aerial vehicles (UAVs) can be used as aerial wireless base stations when cellular networks go down. Prior studies on UAV-based wireless coverage typically consider downlink scenarios from an aerial base station to ground users. In this paper, we consider an uplink scenario under disaster situations (such as earthquakes or floods), when cellular networks are down. We formulate the problem of optimal UAV placement, where the objective is to determine the placement of a single UAV such that the sum of time durations of uplink transmissions is maximized. We prove that the constraint sets of problem can be represented by the intersection of half spheres and the region formed by this intersection is a convex set in terms of two variables. This proof enables us to transform our problem to an optimization problem with two variables. We also prove that the objective function of the transformed problem is a concave function under a restriction on the minimum altitude of the UAV and propose a gradient projection-based algorithm to find the optimal location of the UAV. We validate the analysis by simulations and demonstrate the effectiveness of the proposed algorithm under different cases.
\end{abstract}

Index Terms-Unmanned aerial vehicles, lifetime of wireless network, emergency response, convex optimization, gradient projection algorithm.

\section{INTRODUCTION}

UAVs can be used to provide wireless coverage during emergency cases where each UAV serves as an aerial wireless base station when the cellular network goes down [1]. They can also be used to supplement the ground base station in order to provide better coverage and higher data rates for the users [2].

Prior studies on UAV-based wireless coverage typically consider downlink scenarios from a UAV to ground users. The authors in [3] investigate the downlink coverage performance of a UAV, where the objective is to find the optimal UAV altitude which leads to the maximum ground coverage and the minimum transmit power. In [4], the authors consider the downlink scenario, where the goal is to minimize the total required transmit power of UAVs while satisfying the users rate requirements. In [5]-[8], the authors propose using a UAV to provide wireless coverage for users during emergency cases and special events. Due to the limited transmit power of the UAV, the authors in [9] study the problem of minimizing the number of UAVs required to cover the indoor users.

Only few studies consider the uplink scenario in which the ground wireless devices transmit data to a UAV. The authors in [10] study the throughput maximization problem in UAV relaying systems by optimizing the source/relay transmit power along with the UAV trajectory, subject to practical mobility constraints. In [11], the authors present a UAV enabled data collection system, where a UAV is dispatched to collect a given amount of data from ground terminals at fixed location. They aim to find the optimal ground terminal transmit power and
UAV trajectory that achieve different Pareto optimal energy trade-offs between the ground terminal and the UAV.

Under disaster situations (such as earthquakes or floods), users may not be able to communicate with remote-undamaged terrestrial ground stations due to the limited transmit power of wireless devices. They are also not able to recharge their wireless devices due to physical damage to energy infrastructure. In the case of Hurricane Katrina, about 700,000 customers in Louisiana and almost 200,000 in Mississippi lost power [12]. In such situations, providing wireless coverage becomes more important, since people in the disaster area seek information for themselves to learn about the emergency event, locate their family and friends, and report their safety [13], [14]. In this paper, we are motivated to explore if the placement of UAV can enhance the time durations of uplink transmissions of wireless devices. To the best of our knowledge, this is the first work that proposes using a UAV to maximize the sum of time durations of uplink transmissions under disaster situations, where the ground users are not able to recharge their wireless devices due to physical damage to energy infrastructure. We summarize our main contributions as follows. First, we formulate the problem of optimal UAV placement, where the objective is to maximize the lifetime of wireless devices, the lifetime represents the sum of time durations of uplink transmissions. Second, we prove that the constraint sets of problem can be represented by the intersection of half spheres and the region formed by this intersection is a convex set in terms of two variables. This proof enables us to transform our problem into an optimization problem with two variables. Third, we prove that the objective function of the transformed problem is a concave function under a restriction on the minimum altitude of UAV and propose a gradient projection-based algorithm to find the optimal location of UAV.

The rest of this paper is organized as follows. In Section II, we describe the system model. In Section III, we formulate the problem of UAV placement with an objective of maximizing the sum of time durations of uplink transmissions and present a gradient projection-based algorithm to find the optimal location of the UAV under a restriction on the minimum altitude. Finally, we present our numerical results in Section IV and make concluding remarks in Section V.

\section{SYSTEM MODEL}

Let $\left(X_{u}, Y_{u}, Z_{u}\right)$ denote the 3D location of the UAV. We assume that $|I|$ ground users are located according to a probability distribution $f(x, y)$. Each user $i \in I$ has a wireless device with residual energy $E_{i}$ and the maximum transmit power of each device is $P_{\max }$. The users must be served by a single UAV that acts as aerial base station to collect data from users as shown in Figure 1 We consider an uplink 


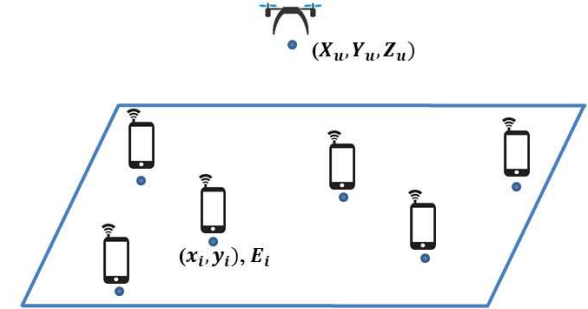

Fig. 1: Ground users transmitting data to a UAV

scenario in which the ground users adopt a frequency division multiple access (FDMA) technique to transmit data to a UAV at a desired data rate $R$. FDMA allocates one subchannel to each user for communications and hence the channels do not interfere with one another. We also assume that each user $i \in I$ is served by a UAV for a time $\tau_{i}$ seconds and this time depends on the residual energy of wireless device represented by the battery level $E_{i}$. The time duration of uplink transmission $\tau_{i}$ must be greater than or equal to $\tau_{t h}$.

In this paper, we assume that the wireless channel between ground user $i$ and UAV is line of sight dominated, so that the free space path loss model is adopted similar to [10] and [11]. The path loss is given as follows:

$$
L_{i}=\left(\frac{4 \pi d_{i} f}{c}\right)^{2}
$$

where $d_{i}=\sqrt{\left(X_{u}-x_{i}\right)^{2}+\left(Y_{u}-y_{i}\right)^{2}+\left(Z_{u}\right)^{2}}$ is the distance between ground user $i$ and a UAV, $f$ is frequency (in $\mathrm{Hz}$ ) and $c$ is the speed of light (in $\mathrm{m} / \mathrm{s}$ ). Notice that when the distance between a ground user and UAV (i.e., $d_{i}$ ) increases, the transmit power (i.e., $p_{i}$ ) increases, while the time duration of uplink transmission (i.e., $\tau_{i}$ ) decreases.

\section{PROBLEM FORMULATION}

Consider a transmission between a user located at $\left(x_{i}, y_{i}\right)$ and a UAV located at $\left(X_{u}, Y_{u}, Z_{u}\right)$. The rate for user $i$ is given by:

$$
C_{i}=B_{i} \log _{2}\left(1+\frac{p_{i} / L_{i}}{N}\right)
$$

where $B_{i}$ is the transmission bandwidth of user $i, p_{i}$ is the transmit power from user $i$ to the UAV, $L_{i}$ is the path loss between user $i$ and the UAV and $N$ is the noise power.

Let us assume that all users have the same data rate $R$ and each user has a channel with bandwidth equals $B /|I|$, where $B$ is the UAV bandwidth and $|I|$ is the number of ground users. Then, the minimum power required to satisfy this rate for each user is given by:

$$
p_{i}=\left(2^{\frac{R \cdot|I|}{B}}-1\right) \cdot N \cdot L_{i}
$$

Our goal is to find the optimal location of the UAV such that the lifetime of wireless devices defined by $T$ is maximized.
Here, the lifetime $T$ represents the sum of time durations of uplink transmissions. Our problem can be formulated as:

$$
\max _{\left(X_{u}, Y_{u}, Z_{u}\right), \tau_{i}} T=\sum_{i=1}^{|I|} \tau_{i}
$$

subject to

$$
\begin{aligned}
& \left(2^{\frac{R \cdot|I|}{B}}-1\right) \cdot N \cdot L_{i} \leq P_{\max } \quad \forall i \in I \\
& \tau_{i} \geq \tau_{\text {th }} \quad \forall i \in I \\
& \tau_{i} \cdot\left(2^{\frac{R .|I|}{B}}-1\right) \cdot N \cdot L_{i} \leq E_{i} \quad \forall i \in I \\
& x_{\min } \leq X_{u} \leq x_{\max } \\
& y_{\min } \leq Y_{u} \leq y_{\max } \\
& z_{\min } \leq Z_{u} \leq z_{\max }
\end{aligned}
$$

Here, constraint set (4.a) ensures that the transmit power of each wireless device should not exceed its maximum transmit power $P_{\max }$. Constraint set (4.b) guarantees that each ground user $i \in I$ is served by UAV for a time greater than $\tau_{t h}$ seconds. Constraint set (4.c) ensures that the total energy consumed by user's device should not exceed its battery energy level $E_{i}$. Constraints (4.d-4.f) represent the minimum and maximum allowed values for $X_{u}, Y_{u}$ and $Z_{u}$.

From (1), we can notice that the optimal altitude of UAV that maximizes the lifetime of wireless devices is equal to $z_{\text {min }}$, which could correspond to the minimum altitude due to safety consideration [11]. Now, our objective is to find the optimal 2D placement of the UAV such that the lifetime of wireless devices is maximized. Even though the problem has a number of nonlinear constraints, we can transform (4) to an optimization problem with two variables by proving that the constraint sets (4.a-4.c) can be represented by the intersection of half spheres and the region formed by this intersection is a convex set in terms of $\left(X_{u}, Y_{u}\right)$.

Theorem 1. The constraint sets (4.a-4.c) can be represented by the intersection of half spheres and the region formed by this intersection is a convex set in terms of $\left(X_{u}, Y_{u}\right)$.

Proof. From (1) and (3), the transmit power of ground user $i$ is given by:

$$
p_{i}=\left(2^{\frac{R \cdot|I|}{B}}-1\right) \cdot N \cdot\left(\frac{4 \pi d_{i} f}{c}\right)^{2}=K d_{i}^{2}
$$

where $K$ is a constant and equals $\left(2^{\frac{R \cdot|I|}{B}}-1\right) \cdot N \cdot\left(\frac{4 \pi f}{c}\right)^{2}$. Now, to satisfy constraint set (4.a), $p_{i}$ must be less than $P_{\max }$. From (5), the range of distances $d_{1}$ that satisfies the constraint set (4.a) is given by:

$$
d_{1} \leq \sqrt{\frac{P_{\max }}{K}}
$$

The range of distances $d_{1}$ represents a half sphere with radius $\sqrt{\frac{P_{\max }}{K}}$ as shown in Figure 2. To satisfy constraint sets (4.b) and (4.c), $p_{i}$ must be less than $\frac{E_{i}}{\tau_{t h}}$. From (5), the range of distances $d_{2}$ that satisfies constraint sets (4.b) and (4.c) is given by:

$$
d_{2} \leq \sqrt{\frac{E_{i}}{\tau_{t h} K}}
$$




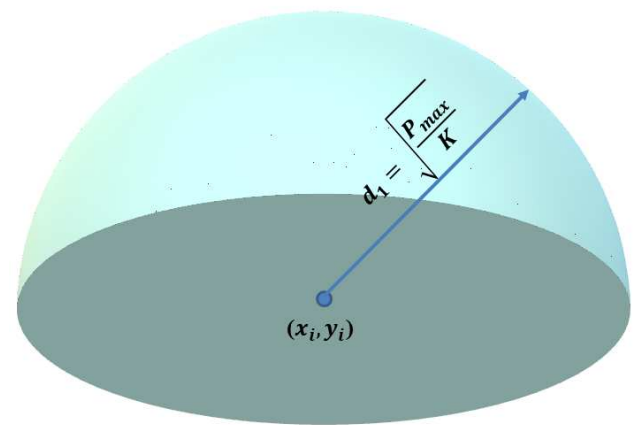

Fig. 2: The range of distances that satisfies constraint set (4.a).

The range of distances $d_{2}$ also represents a half sphere with radius $\sqrt{\frac{E_{i}}{\tau_{t h K} K}}$ as shown in Figure 3. For each ground user $i$, the transmit power $p_{i}$ and the time duration of uplink transmission $\tau_{i}$ that satisfy the constraint sets (4.a)-(4.c) can be represented by a half sphere with radius:

$$
\min \left\{\sqrt{\frac{P_{\max }}{K}}, \sqrt{\frac{E_{i}}{\tau_{t h} K}}\right\}
$$

The half sphere is a convex set and the intersection of convex sets is also a convex [15].

From Theorem 1, we can represent the transmit power $p_{i}$ and the time duration of uplink transmission $\tau_{i}$ constraints for each user as a half sphere. The intersection of all half spheres represents the convex set $V$ that satisfies the constraint sets (4.a)-(4.c) for all users. Therefore, we restrict the placement of UAV to be in $V$. Now, we can transform our problem to an optimization problem with two variables $\left(X_{u}, Y_{u}\right)$, where $\left(X_{u}, Y_{u}\right)$ represent the 2D placement of UAV. The proposed algorithm to find the convex set $V$ is shown in Algorithm 1 as follows: The inputs are the locations of users, the maximum transmit power, the energy of each wireless device, the data rate, the total bandwidth, the operating frequency, the noise power and the threshold time duration of uplink transmission. In steps (10-12), we find the range of distances that satisfies the maximum power constraint. On the other hand, steps (1316) find the range of distances that satisfies the threshold time duration of uplink transmission. In steps (17-21), we find the convex set $V$.

Now, the objective function in (4) can be represented as:

$$
\begin{gathered}
\sum_{i=1}^{|I|} \tau_{i}=\sum_{i=1}^{|I|} \frac{E_{i}}{p_{i}}=\sum_{i=1}^{|I|} \frac{E_{i}}{\left(2^{\frac{R \cdot|I|}{B}}-1\right) \cdot N \cdot L_{i}}= \\
\sum_{i=1}^{|I|} \frac{E_{i}}{\left(2^{\frac{R .|I|}{B}}-1\right) \cdot N \cdot\left(\frac{4 \pi d_{i} f}{c}\right)^{2}}=\sum_{i=1}^{|I|} \frac{E_{i}}{K d_{i}^{2}}
\end{gathered}
$$

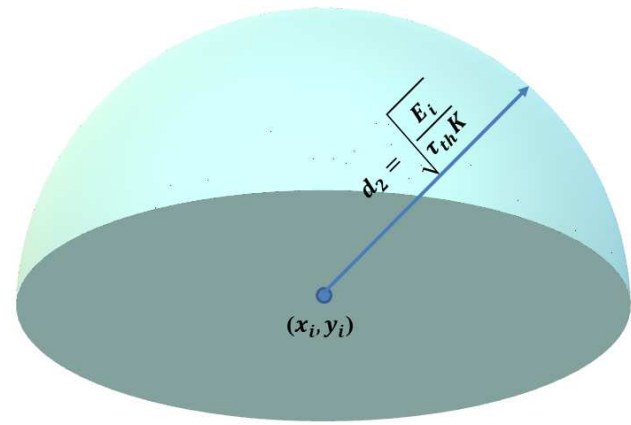

Fig. 3: The range of distances that satisfies constraint sets (4.b) and (4.c).

Since $K$ is constant, our problem can be formulated as:

$$
\begin{aligned}
& \max _{\left(X_{u}, Y_{u}\right)} \sum_{i=1}^{|I|} \frac{E_{i}}{\left(X_{u}-x_{i}\right)^{2}+\left(Y_{u}-y_{i}\right)^{2}+z_{\text {min }}^{2}} \\
& \text { subject to } \\
& \left(X_{u}, Y_{u}\right) \in \\
& \bigcap_{i=1}^{|I|}\left\{\left(X_{u}, Y_{u}\right) \in \mathbf{R}^{2} \mid \sqrt{\left(X_{u}-x_{i}\right)^{2}+\left(Y_{u}-y_{i}\right)^{2}+z_{\text {min }}^{2}} \leq d_{i}\right\}
\end{aligned}
$$

The transformed problem (10) is equivalent to problem (4). 
In the next theorem, we prove that the objective function is concave under a restriction on the minimum altitude of UAV $z_{\text {min }}$. This theorem enables us to find the optimal $\left(X_{u}, Y_{u}\right)$ placement for UAV.

Theorem 2. The objective function of (10) is concave if the minimum altitude of $U A V z_{\text {min }}$ is greater than $\sqrt{3} d_{\text {max }}$.

Proof. We know that the nonnegative weighted sums preserve the concavity of function [15]. Since $E_{i}>0, \forall i \in I$, we need to prove that (11) is a concave function.

$$
f=\frac{1}{\left(X_{u}-x_{i}\right)^{2}+\left(Y_{u}-y_{i}\right)^{2}+z_{\text {min }}^{2}}, \forall i \in I
$$

Using the second order condition, the function $f$ is concave if the Hessian is negative semidefinite [15]. Now, the Hessian is negative semidefinite if we satisfy these conditions:

$$
\begin{aligned}
& \text { (a) } \frac{d^{2} f}{d X_{u}^{2}} \leq 0, \quad \forall i \in I \\
& \text { (b) } \frac{d^{2} f}{d Y_{u}^{2}} \leq 0, \quad \forall i \in I \\
& \text { (c) } \frac{d^{2} f}{d X_{u}^{2}} \frac{d^{2} f}{d Y_{u}^{2}}-\left(\frac{d^{2} f}{d X d Y_{u}}\right)^{2} \geq 0, \quad \forall i \in I
\end{aligned}
$$

To check the first condition, we need to find $\frac{d^{2} f}{d X_{u}^{2}}$ :

$$
\begin{aligned}
& \frac{d f}{d X_{u}}=\frac{-2\left(X_{u}-x_{i}\right)}{\left(\left(X_{u}-x_{i}\right)^{2}+\left(Y_{u}-y_{i}\right)^{2}+z_{\text {min }}^{2}\right)^{2}} \\
& \frac{d^{2} f}{d X_{u}^{2}}=\frac{-2\left(\left(X_{u}-x_{i}\right)^{2}+\left(Y_{u}-y_{i}\right)^{2}+z_{\text {min }}^{2}\right)^{2}}{\left(\left(X_{u}-x_{i}\right)^{2}+\left(Y_{u}-y_{i}\right)^{2}+z_{\text {min }}^{2}\right)^{4}}+ \\
& \frac{8\left(X_{u}-x_{i}\right)^{2}\left(\left(X_{u}-x_{i}\right)^{2}+\left(Y_{u}-y_{i}\right)^{2}+z_{\text {min }}^{2}\right)}{\left(\left(X_{u}-x_{i}\right)^{2}+\left(Y_{u}-y_{i}\right)^{2}+z_{\text {min }}^{2}\right)^{4}} \\
& =\frac{-2\left(\left(X_{u}-x_{i}\right)^{2}+\left(Y_{u}-y_{i}\right)^{2}+z_{\text {min }}^{2}\right)+8\left(X_{u}-x_{i}\right)^{2}}{\left(\left(X_{u}-x_{i}\right)^{2}+\left(Y_{u}-y_{i}\right)^{2}+z_{\text {min }}^{2}\right)^{3}} \\
& =\frac{6\left(X_{u}-x_{i}\right)^{2}-2\left(Y_{u}-y_{i}\right)^{2}-2 z_{\text {min }}^{2}}{\left(\left(X_{u}-x_{i}\right)^{2}+\left(Y_{u}-y_{i}\right)^{2}+z_{\text {min }}^{2}\right)^{3}}
\end{aligned}
$$

From (13), $\frac{d^{2} f}{d X_{u}^{2}} \leq 0, \forall i \in I$ if:

$$
z_{\text {min }}^{2}>3\left(X_{u}-x_{i}\right)^{2}-\left(Y_{u}-y_{i}\right)^{2}, \forall i \in I
$$

Similarly, $\frac{d^{2} f}{d Y_{u}^{2}} \leq 0, \forall i \in I$ if:

$$
z_{\text {min }}^{2}>3\left(Y_{u}-y_{i}\right)^{2}-\left(X_{u}-x_{i}\right)^{2}, \forall i \in I
$$

To check the third condition, we need to find $\frac{d^{2} f}{d X_{u}^{2}} \frac{d^{2} f}{d Y_{u}^{2}}-$ $\left(\frac{d^{2} f}{d X d Y_{u}}\right)^{2}$

$$
\frac{d^{2} f}{d X_{u} d Y_{u}}=\frac{8\left(X_{u}-x_{i}\right)\left(Y_{u}-y_{i}\right)}{\left(\left(X_{u}-x_{i}\right)^{2}+\left(Y_{u}-y_{i}\right)^{2}+z_{\text {min }}^{2}\right)^{3}}
$$

From (16), we get:

$$
\begin{aligned}
& \frac{d^{2} f}{d X_{u}^{2}} \frac{d^{2} f}{d Y_{u}^{2}}-\left(\frac{d^{2} f}{d X d Y_{u}}\right)^{2}= \\
& \left(\frac{-2\left(\left(X_{u}-x_{i}\right)^{2}+\left(Y_{u}-y_{i}\right)^{2}+z_{\text {min }}^{2}\right)+8\left(X_{u}-x_{i}\right)^{2}}{\left(\left(X_{u}-x_{i}\right)^{2}+\left(Y_{u}-y_{i}\right)^{2}+z_{\text {min }}^{2}\right)^{3}} \times\right. \\
& \left.\frac{-2\left(\left(X_{u}-x_{i}\right)^{2}+\left(Y_{u}-y_{i}\right)^{2}+z_{\text {min }}^{2}\right)+8\left(Y_{u}-y_{i}\right)^{2}}{\left(\left(X_{u}-x_{i}\right)^{2}+\left(Y_{u}-y_{i}\right)^{2}+z_{\text {min }}^{2}\right)^{3}}\right)- \\
& \frac{64\left(X_{u}-x_{i}\right)^{2}\left(Y_{u}-y_{i}\right)^{2}}{\left(\left(X_{u}-x_{i}\right)^{2}+\left(Y_{u}-y_{i}\right)^{2}+z_{\text {min }}^{2}\right)^{6}}= \\
& \frac{4\left(\left(X_{u}-x_{i}\right)^{2}+\left(Y_{u}-y_{i}\right)^{2}+z_{\text {min }}^{2}\right)^{2}}{\left(\left(X_{u}-x_{i}\right)^{2}+\left(Y_{u}-y_{i}\right)^{2}+z_{m i n}^{2}\right)^{6}}- \\
& \frac{16\left(Y_{u}-y_{i}\right)^{2}\left(\left(X_{u}-x_{i}\right)^{2}+\left(Y_{u}-y_{i}\right)^{2}+z_{\text {min }}^{2}\right)}{\left(\left(X_{u}-x_{i}\right)^{2}+\left(Y_{u}-y_{i}\right)^{2}+z_{\text {min }}^{2}\right)^{6}}- \\
& \frac{16\left(X_{u}-x_{i}\right)^{2}\left(\left(X_{u}-x_{i}\right)^{2}+\left(Y_{u}-y_{i}\right)^{2}+z_{\text {min }}^{2}\right)}{\left(\left(X_{u}-x_{i}\right)^{2}+\left(Y_{u}-y_{i}\right)^{2}+z_{\text {min }}^{2}\right)^{6}}+ \\
& \frac{64\left(X_{u}-x_{i}\right)^{2}\left(Y_{u}-y_{i}\right)^{2}}{\left(\left(X_{u}-x_{i}\right)^{2}+\left(Y_{u}-y_{i}\right)^{2}+z_{m i n}^{2}\right)^{6}}- \\
& \frac{64\left(X_{u}-x_{i}\right)^{2}\left(Y_{u}-y_{i}\right)^{2}}{\left(\left(X_{u}-x_{i}\right)^{2}+\left(Y_{u}-y_{i}\right)^{2}+z_{\text {min }}^{2}\right)^{6}}= \\
& \frac{4\left(\left(X_{u}-x_{i}\right)^{2}+\left(Y_{u}-y_{i}\right)^{2}+z_{\text {min }}^{2}\right)}{\left(\left(X_{u}-x_{i}\right)^{2}+\left(Y_{u}-y_{i}\right)^{2}+z_{\text {min }}^{2}\right)^{5}}+ \\
& \frac{-16\left(X_{u}-x_{i}\right)^{2}-16\left(Y_{u}-y_{i}\right)^{2}}{\left(\left(X_{u}-x_{i}\right)^{2}+\left(Y_{u}-y_{i}\right)^{2}+z_{\text {min }}^{2}\right)^{5}}= \\
& \frac{-12\left(X_{u}-x_{i}\right)^{2}-12\left(Y_{u}-y_{i}\right)^{2}+4 z_{\text {min }}^{2}}{\left(\left(X_{u}-x_{i}\right)^{2}+\left(Y_{u}-y_{i}\right)^{2}+z_{\text {min }}^{2}\right)^{5}} \\
& \text { From (17), } \frac{d^{2} f}{d X_{u}^{2}} \frac{d^{2} f}{d Y_{u}^{2}}-\left(\frac{d^{2} f}{d X d Y_{u}}\right)^{2} \geq 0, \forall i \in I \text { if: } \\
& z_{\text {min }}^{2}>3\left(X_{u}-x_{i}\right)^{2}+3\left(Y_{u}-y_{i}\right)^{2}, \forall i \in I
\end{aligned}
$$

From (14), (15) and (18), the Hessian is negative semidefinite if we satisfy these conditions:

$$
\begin{aligned}
& (a) z_{\text {min }}^{2}>3\left(X_{u}-x_{i}\right)^{2}-\left(Y_{u}-y_{i}\right)^{2}, \forall i \in I \\
& (b) z_{\text {min }}^{2}>3\left(Y_{u}-y_{i}\right)^{2}-\left(X_{u}-x_{i}\right)^{2}, \forall i \in I \\
& (c) z_{\text {min }}^{2}>3\left(X_{u}-x_{i}\right)^{2}+3\left(Y_{u}-y_{i}\right)^{2}, \forall i \in I
\end{aligned}
$$

From the three conditions in (19), we can notice that if we satisfy condition (c), we then satisfy conditions (a) and (b). Let us define $d_{\max }$ as a maximum possible $2 \mathrm{D}$ distance in the geographical area (i.e., if the users are distributed in a circular geographical area, then $d_{\max }$ is equal to the diameter of circle). From condition (c), if $z_{\min }>\sqrt{3} d_{\max }$ then the objective function of (10) is concave where $d_{\max }>$ $\sqrt{\left(X_{u}-x_{i}\right)^{2}+\left(Y_{u}-y_{i}\right)^{2}}, \forall i \in I$. Here, we can notice that the altitude of UAV $z_{\min }$ controls the concavity of the objective function.

Theorem 2 enables us to find the optimal $\left(X_{u}, Y_{u}\right)$ placement for UAV, when the altitude of UAV $z_{\text {min }}$ is greater than $\sqrt{3} d_{\max }$, which is a practical altitude especially for high altitude platforms. In order to find this point, we propose the Gradient Projection Algorithm [16]. The gradient algorithm is 


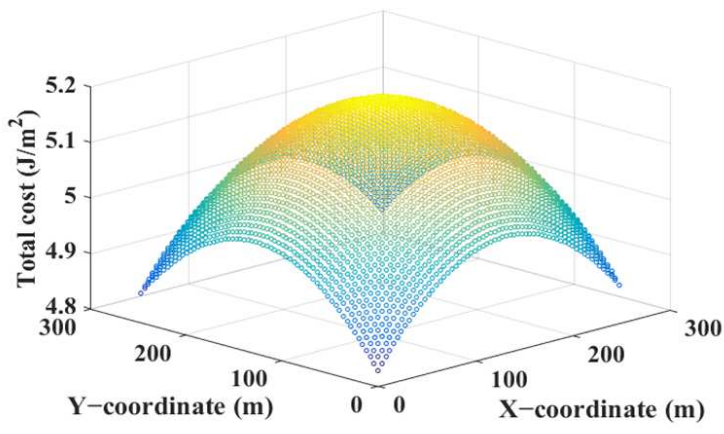

(a) Total cost at 650 meters

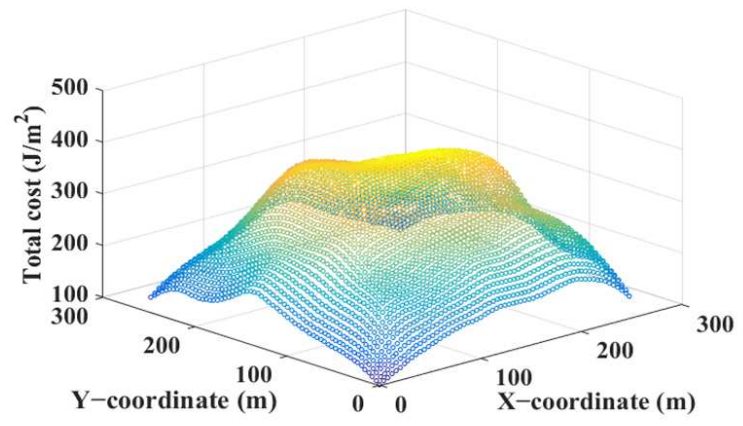

(b) Total cost at 30 meters

Fig. 4: Total cost at different altitudes

not applicable to constrained optimization problems, because even if we start inside the feasible region, an update can take us outside that region. A simple way to solve this problem is to project back to the feasible region whenever such a situation arises. The gradient projection algorithm is given by:

$$
\left.\left(X_{u}, Y_{u}\right)^{n+1}=\left[\left(X_{u}, Y_{u}\right)^{n}+\gamma \cdot \nabla F\left(\left(X_{u}, Y_{u}\right)^{n}\right)\right)\right]^{+} .
$$

Here, $n$ is the iteration number, $\gamma$ is a positive step size, $\nabla F$ is the gradient of the objective function in (10) and $[q]^{+}$denotes the orthogonal projection of vector $q$ onto convex set $Q$. In particular, $[q]^{+}$is defined by: .

$$
[q]^{+}=\arg \min _{w \in Q}\|w-q\|_{2}
$$

To find the orthogonal projection, we use the matlab function fmincon. The pseudo code of the gradient projection algorithm is shown in Algorithm 2.

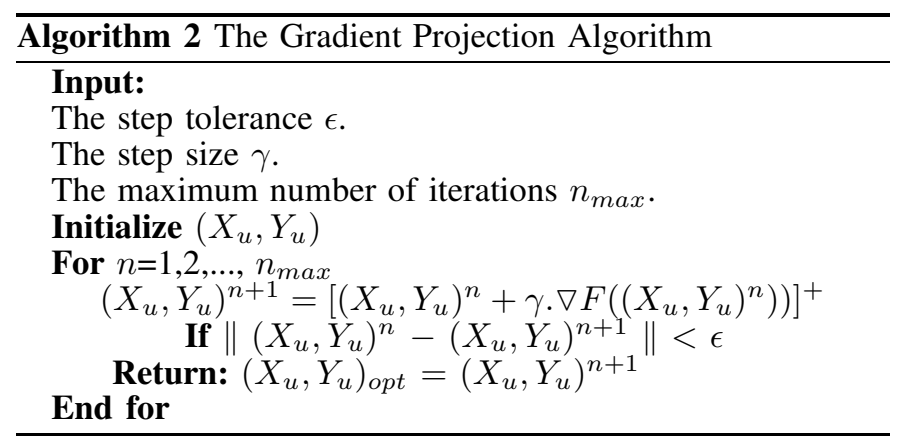

\section{NUMERICAL RESULTS}

In this section, we first verify the results of Theorem 2, then we use the gradient projection algorithm to find the optimal placement for UAV under different cases. Table I lists the parameters used in the numerical analysis.

To verify the results of Theorem 2, we assume that 200 ground users are uniformly distributed in a geographical area of size $250 \mathrm{~m} \times 250 \mathrm{~m}$, then we plot the objective function in (10) without any constraints at two different altitudes of the $\mathrm{UAV}$. The first value for altitude $z_{\min }$ is 650 meters, which is greater than $\sqrt{3} d_{\max }$ and satisfies the condition in Theorem 2 . The second value for altitude is 30 meters and it does not satisfy the condition in Theorem 2. In Figure 4.a, we can notice that the objective is concave when the altitude of UAV is equal to 650 meters. On the other hand, the objective function
TABLE I: Parameters in numerical analysis

\begin{tabular}{|c|c|}
\hline Dimensions of area & {$[0,250] \times[0,250]$} \\
\hline Number of ground users & 200 users \\
\hline Maximum number of iterations $n_{\max }$ & 100 \\
\hline Maximum transmit power of wireless device $P_{\max }$ & $0.5 \mathrm{watt}$ \\
\hline Energy of each wireless device $E_{i}$ in joule & $4500+13500^{*} \mathrm{rand}(200,1)$ \\
\hline Data rate $R$ & $4 \mathrm{Mbps}$ \\
\hline Total bandwidth $B$ & $50 \mathrm{MHz}$ \\
\hline The noise power $N$ & $1 \times 10^{-14}$ \\
\hline The carrier frequency $f$ & $4 \mathrm{Ghz}$ \\
\hline Threshold time duration of uplink transmission $\tau_{t h}$ & $900 \mathrm{~seconds}$ \\
\hline Minimum altitude for UAV & $650 \mathrm{~meters}$ \\
\hline
\end{tabular}

becomes non-concave at 30 meters as shown in Figure 4.b. We can also notice that the objective function at low altitude has better costs compared to results at high altitude, which make our approach practical for UAVs that have high altitude constraints like Project Loon [17] by Google .

In Figure 5, we place the UAV at 650 meters and use the gradient projection algorithm to find the optimal 2D placement that maximizes the lifetime of wireless devices when the ground users are uniformly distributed. The optimal placement for UAV is $(131,128,650)$ and the optimal cost is 5.19 $J / m^{2}$ (282096 seconds). We can notice that the projection of the optimal point is located near the center of deployment region. This is because the devices that have minimum costs are located at the corners of the deployment region and placing the projection of UAV near the center maximizes the total cost. In Figure 6, we use the gradient projection algorithm to find the optimal 2D placement when the ground users are non-uniformly distributed. The optimal placement for UAV is $(92,156,650)$ and the optimal cost is $5.22 \mathrm{~J} / \mathrm{m}^{2}$ (283727 seconds). We can notice that the placement of UAV is near the high density region.

\section{CONCLUSION}

In this paper, we study the problem of optimal UAV placement, where the objective is to determine the placement of a single UAV such that the sum of time durations of uplink transmissions is maximized. We prove that the constraint sets of problem can be represented by the intersection of half spheres and the region formed by this intersection is a convex set in terms of two variables. This proof enables us to transform our problem to an optimization problem with two variables. We also prove that the objective function of the transformed problem is a concave function under a restriction 


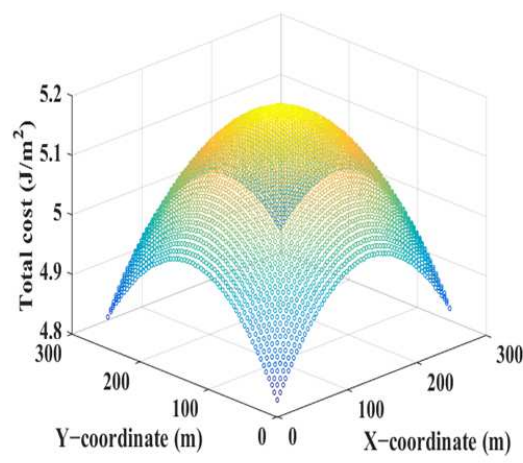

(a) Total cost

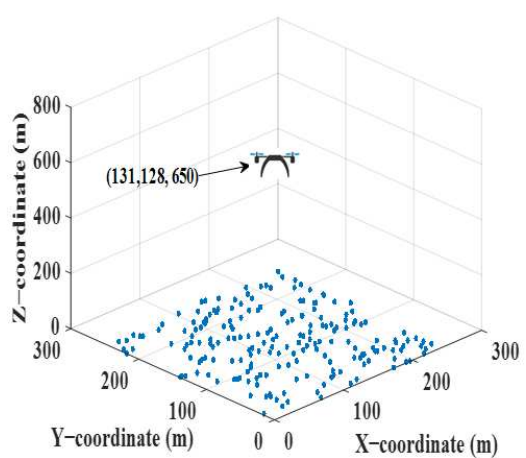

(b) 3D placement of UAV

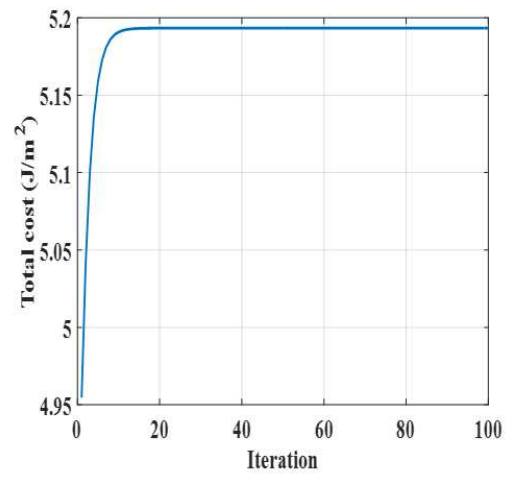

(c) Convergence speed of algorithm

Fig. 5: Simulation results of the uniform distribution case

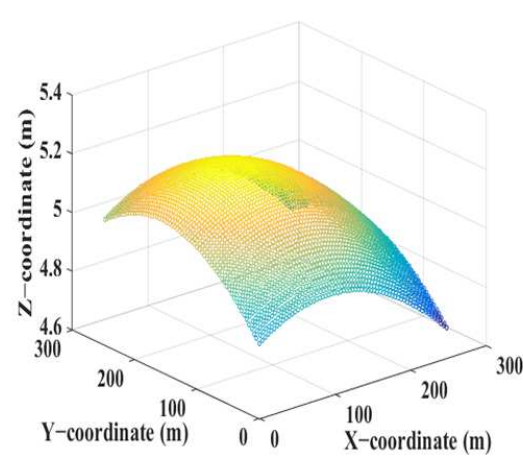

(a) Total cost

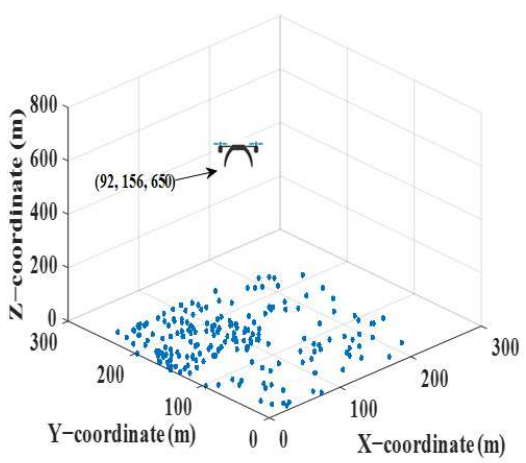

(b) 3D placement of UAV

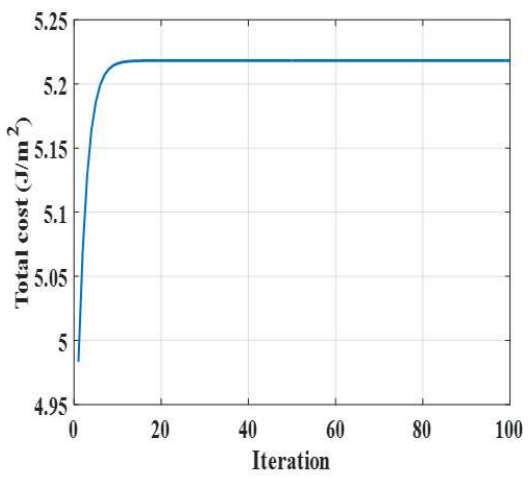

(c) Convergence speed of algorithm

Fig. 6: Simulation results of the non-uniform distribution case

on the minimum altitude of the UAV and propose a gradient projection-based algorithm to find the optimal location of the UAV. As future work, we will study the lifetime maximization problem when multiple UAVs are utilized.

\section{ACKNOWLEDGMENT}

This work was supported in part by the US NSF grants CNS-1647170 and EEC-1560131.

\section{REFERENCES}

[1] H. Shakhatreh, A. Khreishah, J. Chakareski, H. B. Salameh, and I. Khalil, "On the continuous coverage problem for a swarm of uavs," in Sarnoff Symposium, 2016 IEEE 37th. IEEE, 2016, pp. 130-135.

[2] R. I. Bor-Yaliniz, A. El-Keyi, and H. Yanikomeroglu, "Efficient 3-d placement of an aerial base station in next generation cellular networks," in Communications (ICC), 2016 IEEE International Conference on. IEEE, 2016, pp. 1-5.

[3] M. Mozaffari, W. Saad, M. Bennis, and M. Debbah, "Drone small cells in the clouds: Design, deployment and performance analysis," in Global Communications Conference (GLOBECOM), 2015 IEEE. IEEE, 2015, pp. $1-6$.

[4] M. Mozaffari, W. Saad, M. Bennis, and M. Debbah, "Optimal transport theory for power-efficient deployment of unmanned aerial vehicles," in Communications (ICC), 2016 IEEE International Conference on. IEEE, 2016, pp. 1-6.

[5] H. Shakhatreh, A. Khreishah, and B. Ji, "Providing wireless coverage to high-rise buildings using uavs," in 2017 IEEE International Conference on Communications (ICC), May 2017, pp. 1-6.

[6] H. Shakhatreh, A. Khreishah, A. Alsarhan, I. Khalil, A. Sawalmeh, and N. S. Othman, "Efficient 3d placement of a uav using particle swarm optimization," in Information and Communication Systems (ICICS), 2017 8th International Conference on. IEEE, 2017, pp. 258-263.
[7] H. Shakhatreh, A. Khreishah, N. S. Othman, and A. Sawalmeh, "Maximizing indoor wireless coverage using uavs equipped with directional antennas," in Communications (MICC), 2017 IEEE 13th Malaysia International Conference on. IEEE, 2017, pp. 175-180.

[8] A. Sawalmeh, N. S. Othman, H. Shakhatreh, and A. Khreishah, "Providing wireless coverage in massively crowded events using uavs," in Communications (MICC), 2017 IEEE 13th Malaysia International Conference on. IEEE, 2017, pp. 158-163.

[9] H. Shakhatreh, A. Khreishah, and I. Khalil, "The indoor mobile coverage problem using uavs," arXiv preprint arXiv:1705.09771, 2017.

[10] Y. Zeng, R. Zhang, and T. J. Lim, "Throughput maximization for mobile relaying systems," in Globecom Workshops (GC Wkshps), 2016 IEEE. IEEE, 2016, pp. 1-6.

[11] D. Yang, Q. Wu, Y. Zeng, and R. Zhang, "Energy trade-off in ground-to-uav communication via trajectory design," arXiv preprint arXiv:1709.02975, 2017.

[12] A. Kwasinski, P. L. Chapman, P. Krein, W. Weaver et al., "Hurricane katrina: damage assessment of power infrastructure for distribution, telecommunication, and backup." Grainger Center for Electric Machinery and Electromechanics. Technical Report., 2006.

[13] T. Taniguchi, Y. Karasawa, and N. Nakajima, "Effect of coordinated base station in uplink transmission under disaster cells," in Mobile and Wireless Networking (iCOST), 2012 International Conference on Selected Topics in. IEEE, 2012, pp. 83-88.

[14] T. Simon, A. Goldberg, and B. Adini, "Socializing in emergenciesa review of the use of social media in emergency situations," International Journal of Information Management, vol. 35, no. 5, pp. 609-619, 2015.

[15] S. Boyd and L. Vandenberghe, Convex optimization. Cambridge university press, 2004.

[16] D. P. Bertsekas and J. N. Tsitsiklis, Parallel and distributed computation: numerical methods. Prentice hall Englewood Cliffs, NJ, 1989, vol. 23

[17] S. Levy, "How google will use high-flying balloons to deliver internet to the hinterlands," Wired. Retrieved, vol. 15, 2013. 